\title{
EMI Model of an AC/AC Power Converter
}

\author{
Jordi Espina $^{1}$, Josep Balcells ${ }^{1}$, Antoni Arias ${ }^{1}$, Carlos Ortega $^{2}$ and Nestor Berbel ${ }^{1}$ \\ 1) Universitat Politècnica de Catalunya, 2) Escola Universitària Salesiana de Sarrià \\ Electronic Engineering Department \\ espina@eel.upc.edu
}

\begin{abstract}
This paper deals with the validation of an EMI model to predict electromagnetic interferences (EMI) produced by power converters. A direct AC to AC matrix converter (MC) has been selected as experimental setup for the paper, but the modeling method can be applied to other converter topologies.

The method is based on obtaining a high frequency equivalent circuit, using a combined time and frequency domain approach based on: "EMI source identification $\rightarrow$ propagation path impedance $\rightarrow$ derived disturbance". The advantages of the proposed procedure are the computational time reduction and the lack of convergence problems, which may arise when using pure time domain procedures. The paper is focused on the prediction of common mode (CM) EMI of a matrix converter. The simulation results of this case will permit the calculation of currents which leak through the ground connections. Two different EMI sources are compared: one based on PWM waveforms obtained from MATLAB ${ }^{\circledR}$ model and a second one where these data are obtained experimentally from a MC prototype. This procedure allows the comparison of EMI spectra when using simulated waveforms or real source waveforms.
\end{abstract}

\section{INTRODUCTION}

There are different methods to calculate the conducted EMI generated by power converters, which can be broadly classified in two categories: Field computation methods and equivalent circuit methods. Field computation methods are usually based on Maxwell's equations solved by means of Finite Element Methods (FEM) [1], with different possible approaches: differential methods, such as Finite-Difference Time-Domain (FDTD) or integral methods such as Method of Moments (MoM). On the other hand, equivalent circuit methods may also follow different approaches, namely: Time domain analysis, frequency domain analysis and combined time and frequency domain. Advantages of the time domain methods are high resolution and accuracy. However, their drawbacks are large computational time (large simulation periods and small simulation steps) and possible problems of convergence. Alternatively, frequency domain methods, based on "source-path-victim" method [2-5] introduce a remarkable simplicity in terms of the modeling, which reduces drastically the computational effort required for its simulation. On the other hand, their drawbacks are frequency limitation and that they are valid only for conducted emissions. Combined methods [6], on which this paper is based, integrate time domain and frequency domain methods. Time domain simulation is used for EMI sources identification, combining short time, small steps simulation and long time with wider steps. Frequency domain methods, as in [5] perform the system simulation following the above described procedure "EMI source identification $\rightarrow$ propagation path impedance $\rightarrow$ derived disturbance".

Since the main EMI sources in power electronics come from the converters switching and basically produce conducted emissions, the combined method is very suitable to analyze CM in such applications. Following this approach, in [4] a boost rectifier is analyzed and a complete impedance model is presented. Also, a three phase voltage source inverter (VSI) study is presented in [3], which introduces a simplified model to study the CM of these converters. Another work that studies CM of VSI converter is presented in [5], where not only the VSI itself is modeled but also the cabling, motor and plane ground link. Another frequency method based on "Modular-terminal-method" is presented in [7], which introduces a Norton equivalent circuit as basic noise source. This idea permits a simplification of the noise source model of VSI by changing each phase by a basic Norton circuit. This procedure allows the study of all VSI configurations by only adding/removing Norton circuits depending on the topology.

In [8], CM EMI disturbances are addressed when using $\mathrm{MC}$ fed wind turbines. The MC model is based on the indirect inverter-rectifier and virtual DC link approach.

The present paper presents an EMI model based on circuit of impedances that represents the parasitic impedances and leakage paths which characterize the CM circuit. This model is based on [3] to successfully address the study of CM disturbances in a direct $\mathrm{MC}$ configuration [9]. Using a combined time and frequency domain approach will permit a fast prediction of CM produced by matrix converters.

\section{COMBINED TIME-FREQUENCY METHOD}

As pointed out above, the time-frequency method is based on the proper identification of the "source, path and victim" for its further modeling process. The modeling of conducted EMI is essentially based on the solution over the whole frequency range of interest of an equivalent circuit for $\mathrm{CM}$ and a different circuit for DM, as illustrated in Fig.1.

The noise sources to be introduced in the equivalent circuits come from circuit points with large $d v / d t$, which generate EMI current through parasitic coupling capacitances or from $d i / d t$, which generate voltage sources at parasitic inductances. These represent respectively the CM and DM sources. Propagation paths are modeled by the real circuit impedances plus the parasitic impedances involved in current 

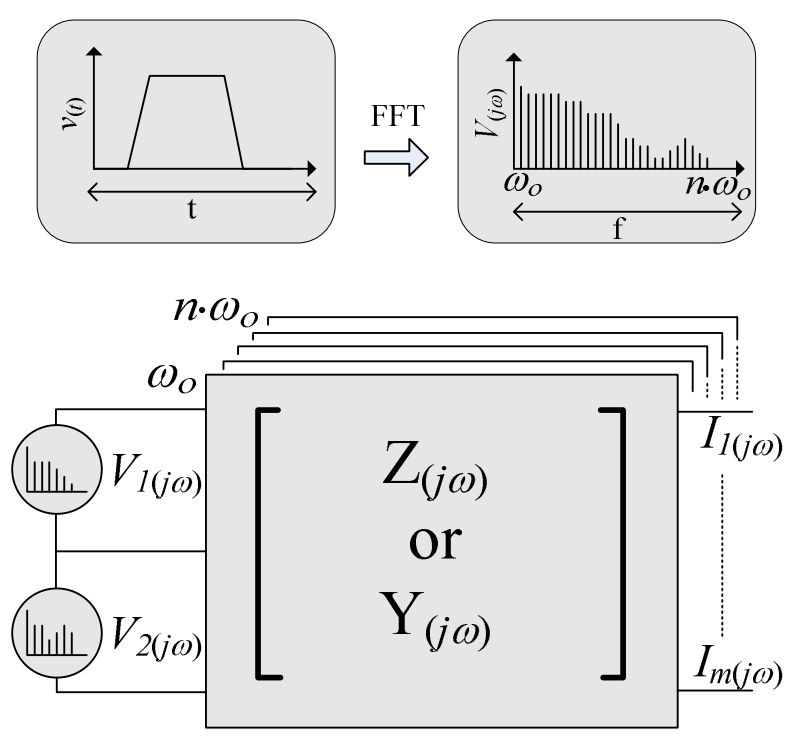

Figure 1 Compact time-Frequency model. Perturbation sources and impedances matrix.

noise circulation between sources and victims. The victims in power electronics are to be found in both sides of the power converter, i.e., electrical grid and load.

Since this paper is focused on the modeling of $\mathrm{CM}$ disturbances, the principal EMI sources are due to fast changes of voltage at certain points of the converter, i.e., large $d v / d t$. In a first step of the simulation, the voltages at such points have been obtained by means of a time domain simulation. To be used later in the frequency domain simulation, a conversion, based on Fast Fourier Transform (FFT), has been performed.

The propagation path impedances to be used in the model have been obtained by means of several measurements performed using impedance analyzer (Fluke PM6306) and vector network analyzer (R\&S ZVRE). The measurements have been done over the entire range of conducted EMI, from $9 \mathrm{kHz}$ to $50 \mathrm{MHz}$. The resulting impedances have been used to fill in a frequency dependent matrix of impedances (or admittances) which is used to solve the circuit model representing the whole system.

Finally, the simulation is performed by solving Kirchhoff's laws in the equivalent circuit, supplied by the sources derived from step 1. The calculations are done for a discrete number of frequencies, which can be distributed following a linear or a logarithmic law. The entire procedure is illustrated in Fig. 1 and can be formulated by Eq. (1), where $n$ is the number of harmonics (number of points of EMI spectrum to be calculated) and $\omega_{o}$ is the base frequency.

$$
i_{\left(n \omega_{o}\right)}=\frac{v_{\left(n \omega_{o}\right)}}{Z_{\left(n \omega_{o}\right)}}
$$

Despite the fact that the overall described process requires a significant number of iterations and time, it's still more effective than the time domain methods because avoid to calculate the long periods of time with small steps.

\section{PLANT DESCRIPTION}

The described method has been applied to obtain the common mode leakage current of a matrix MC drive, which basic High Frequency (HF) equivalent circuit is shown in Fig.2. The system setup used for model validation is formed, at the supply side, by an isolation transformer plus a Line Impedance Stabilization Network (LISN), an input filter and the MC. At the load side of MC there are the cabling and the motor.

The LISN can be used to measure CM and DM disturbances produced by the converter, but in our case it was used just to obtain standard and repeatable test conditions. In fact CM-EMI was measured through a high frequency current probe. The equivalent circuit of the LISN has been modeled as three lumped impedances placed between each of the input phases of $\mathrm{MC}$ filter and ground. These impedances are identified in Fig. 3 as $Z_{S A G}, Z_{S B G}$ and $Z_{S C G}$.

The MC has a low pass input filter, which basically

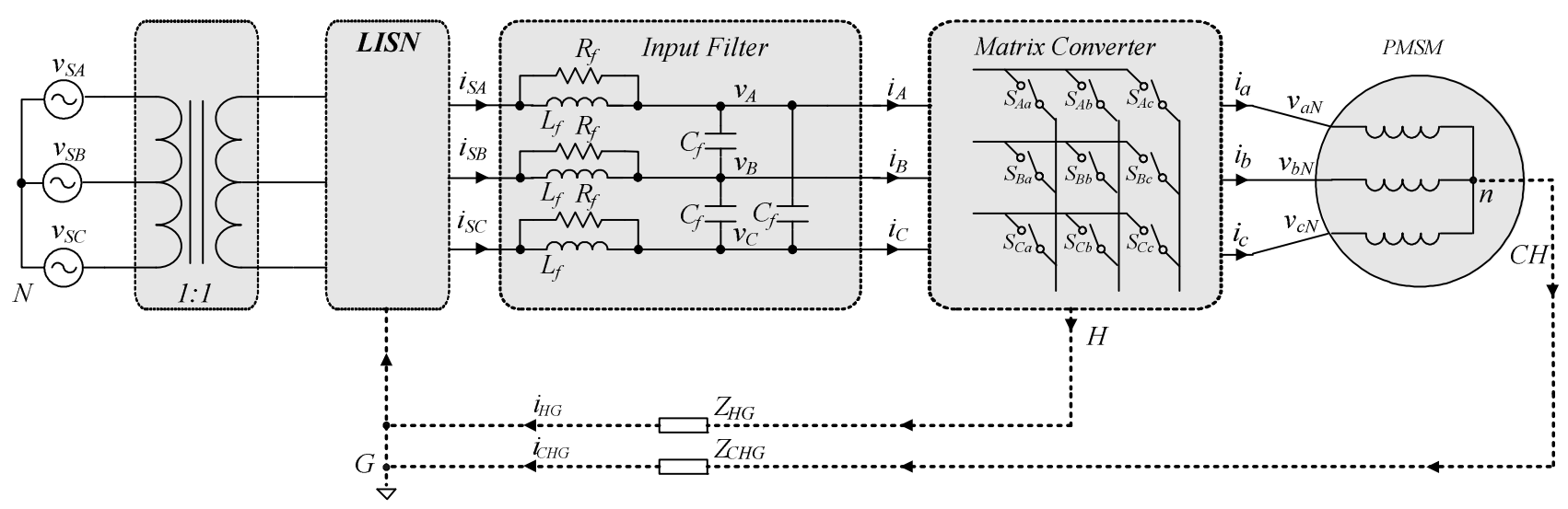

Figure 2. Matrix Converter's set-up and CM paths. 


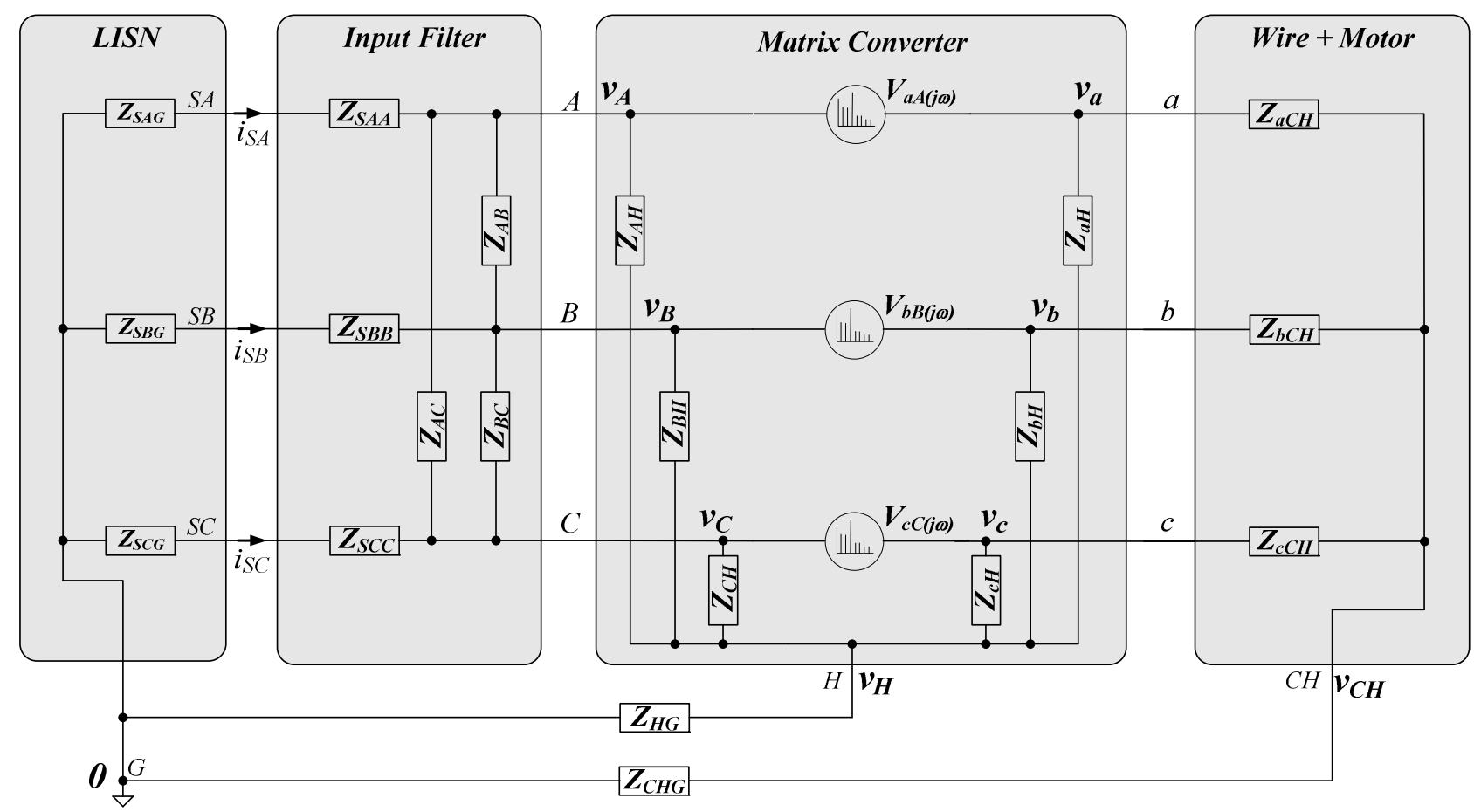

Figure 3. Whole EMI Model set-up; MC, load and LISN.

consists of an $L C$ circuit with a parallel damping resistor as show in Fig.2. The filter has been modeled as two lumped impedances per phase:

1) The serial impedances $\left(Z_{S A A}, Z_{S B B}\right.$ and $\left.Z_{S C C}\right)$, which account for the set $R_{f}, L_{f}$ and the respective HF parasitic impedances.

2) Parallel impedances that appear between phases, which are due to $C_{f}$ capacitors $\left(Z_{A B}, Z_{B C}\right.$ and $\left.Z_{A C}\right)$.

$\mathrm{MC}$ is mainly built by means of an all silicon module that packs all the bidirectional switches. This package reduces the possible parasitic inductances which could come out because of wiring. The characteristic impedances of the module have been measured for each individual phase, as shown and illustrated in Fig.4. The dashed lines and impedances represent the parasitic elements that appear between terminals and ground (mainly heat sink). For example, $Z_{A H}$ in Fig.4 represents the impedance measured between input phase $A$ and ground. The impedances $Z_{B H}$ and $Z_{C H}$ play the same role with regard to the other two phases, $B$ and $C$ respectively. Finally, each output has parasitic impedance which appears between the output terminal and ground. The impedance

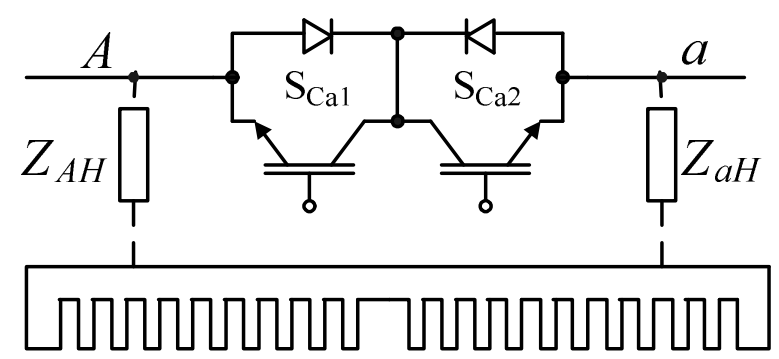

Figure 4. Input and output impedance to heat sink. between phase $a$ and ground is labeled as $Z_{a H}$, and the impedances of phases $\mathrm{b}$ and $\mathrm{c}$ are referenced as $Z_{b H}$ and $Z_{c H}$ respectively. The rest of common impedances between phases can be avoided because the semiconductor module has been modeled as three EMI sources which allow to treat each phase with no dependency between others. All the impedances that are involved in the matrix module have been measured disconnecting the input and output connections from input filter and load respectively.

Another possible path that EMI disturbances flow through ground is the load. In our study case, the load was a permanent magnet synchronous machine (PMSM). For proper EMI modeling, the power transmission cables, the internal motor coupling path and ground cable were characterized.

In Fig. 5, $Z_{\text {wire }}$ and $Z_{\text {ParA }}$ represent the parasitic impedances of the cable, $Z_{L a}$ is the impedance of the motor and $Z_{C M}$ is the coupling impedance between motor winding and ground motor frame [10, 11].

Nevertheless, since the wires were short in the experimental setup, we have simplified the motor and its

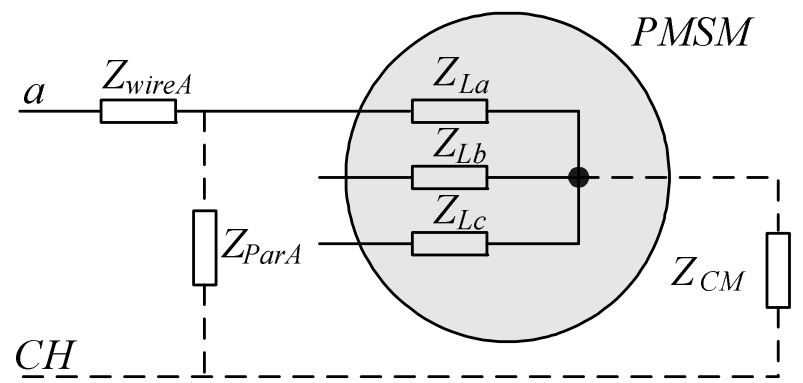

Figure 5. Load impedance model. 


$$
\begin{gathered}
{\left[\begin{array}{c}
0 \\
0 \\
0 \\
0 \\
0 \\
v_{a A} \\
v_{b B} \\
v_{c C}
\end{array}\right]=\left[\begin{array}{c}
\left(\frac{1}{\left(Z_{S A G}+Z_{S A A}\right)}+\frac{1}{Z_{A C}}+\frac{1}{Z_{A B}}+\frac{1}{Z_{A H}}\right)\left(\frac{1}{Z_{a A}}\right. \\
\frac{-1}{Z_{A B}} \\
\frac{1}{Z_{A H}} \\
\frac{1}{Z_{A C}} \\
0 \\
-1 \\
0 \\
0 \\
-\left(\frac{1}{Z_{a H}}+\frac{1}{Z_{A H}}\right) \\
-\left(\frac{1}{Z_{A H}}+\frac{1}{Z_{b H}}\right) \\
\left.+\frac{1}{Z_{a H}}+\frac{1}{Z_{B H}}+\frac{1}{Z_{b H}}+\frac{1}{Z_{c H}}+\frac{1}{Z_{C H}}\right) \\
\left(\frac{1}{Z_{C H}}+\frac{1}{Z_{c H}}\right) \\
0 \\
0 \\
0 \\
0
\end{array}\right.}
\end{gathered}
$$
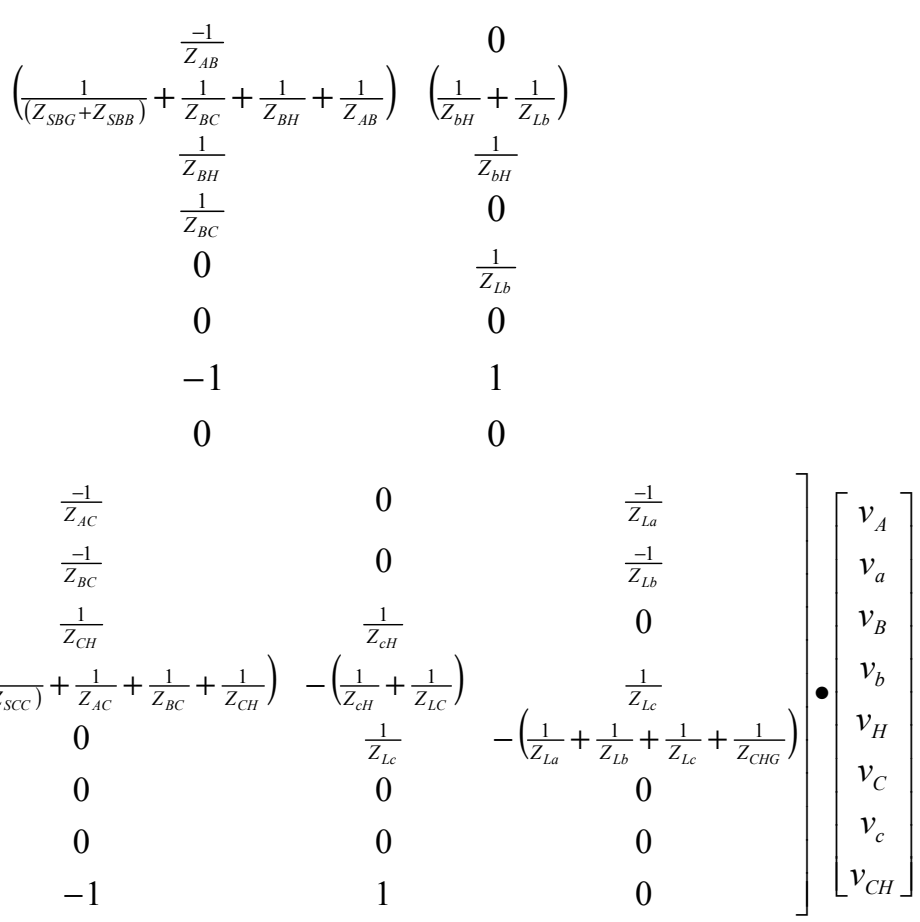

connections to $\mathrm{MC}$ by means of single impedance for each phase $Z_{a C H}, Z_{b C H}$ and $Z_{c C H}$ plus ground impedance that we have been included in $Z_{C H G}$ as it is shown in Fig.3. The impedances $Z_{H G}$ and $Z_{C H G}$ in Fig.3 represent the coupling impedances between heat sink and ground plane and motor stator and ground connection of motor respectively as indicated in Fig.2.

The disturbance sources for the CM model are placed at the points where fast changes of voltage $(d v / d t)$ occur. Usually this happens between each one of the output phase pairs $a-b$, $b-c$ and $c-a$, but this configuration requires modeling the whole semiconductor module. However, this entails increasing the complexity of the model because all the impedances between phases and also the state of each switch must be known. This paper introduces the MC module as a multi-source generation that implies a wide simplification of the model. Three voltage sources between input phases and output phases $\left(v_{a A}, v_{b B}\right.$ and $\left.v_{c C}\right)$ allow modeling the matrix semiconductor module without knowing the internal impedances, IGBTs' characteristics and the others parameters which would make the EMI model unapproachable. This approach is presented and showed in Fig.3. Notice that in many EMI models applied to voltage source inverters (VSI), authors consider the disturbance source to be a voltage source between one of the DC bus terminals (some times mid point of DC bus) and an imaginary mid point or neutral point in the motor. The proposed model does not use such imaginary points, since the DC bus does not exist in a MC and the motor neutral point may not exist if the motor is delta connected.

Fig.3 illustrates the whole CM-EMI equivalent circuit model of the matrix converter proposed in this work. The model combines all the parts described above and shown in Fig.4 and Fig.5.
The model solution simply consists of the network solution using the classical Kirchhoff's laws to obtain the currents in all the circuit branches for each particular frequency. The solution brings to a matrix equation as shown in (2).

\section{RESUlTS}

The proposed model has been used to simulate the system described below, using standard software MATLAB ${ }^{\circledR}$. In order to increase the accuracy of the results the simulation has been divided in two parts: low frequency (from $9 \mathrm{kHz}$ to $1 \mathrm{MHz}$ ) and high frequency (from $1 \mathrm{MHz}$ to $50 \mathrm{MHz}$ ). However, this paper only shows the simulation at low frequencies. The study in the whole frequency range of
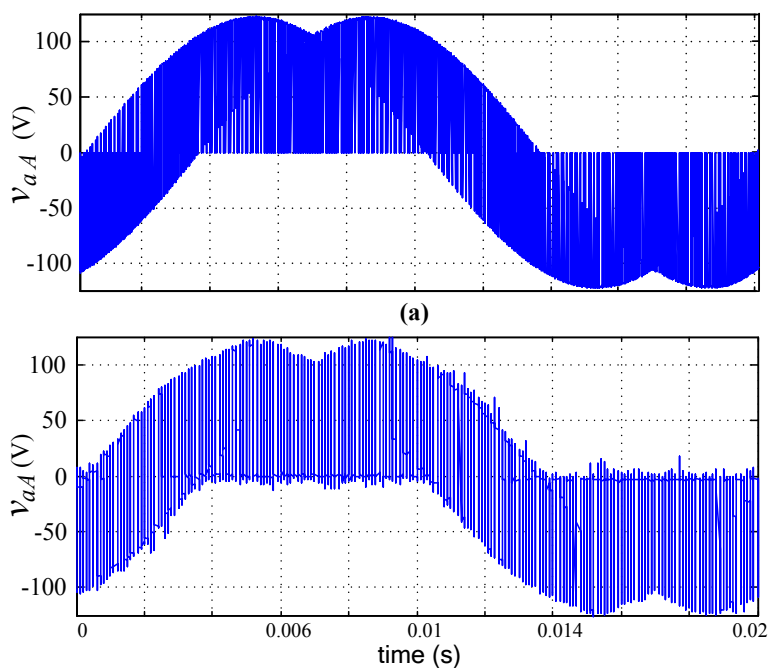

(b)

Fig.6 (a).Simulation wave vaA, (b).Experimental wave vaA 
conducted EMI requires some refinements in the sources model.

The MC drive prototype, used to validate the CM model comprises the following elements:

- Matrix converter prototype: Nottingham University, $7.5 \mathrm{~kW}, 380 \mathrm{~V}$. It is constituted by an IGBT module FM35R12KE3ENG (Eupec).

- Permanent magnet synchronous motor: Control Techniques, $3 \times 220 \mathrm{~V} / 0.94 \mathrm{~kW}$, rated speed 3000rpm.

- LISN: Scwarzbeck Meßelektronik, NNLA8120, $50 \Omega / 5 \Omega+50 \mu \mathrm{H}, 4 \times 25 \mathrm{~A}$.

- Shielded cables: five conductors, section= $1.5 \mathrm{~mm} 2+$ shielding braid.

Every nodal voltage $\left(v_{A}, v_{a}, \ldots, v_{C H}\right)$ and all the branch currents have been evaluated, thus allowing to know the high frequency behavior of the MC. The leakage currents that describe the $\mathrm{CM}$ are the currents flowing through $Z_{H G}$ and $Z_{C H G}$. Therefore the addition of these currents $\left(I_{H G}+I_{C H G}\right)$ is the CM leakage current.

As shown in Fig 6, disturbance CM model voltage sources have been obtained from two different ways: firstly from simulation and secondly from real data captured from the MC prototype.

In a first stage, only ideal voltages were considered, without taking into account the switching transients. In this first approach we have considered ideal switching at constant slew rate. This approximation implies that the source models will only be valid to simulate low frequency disturbances up to approximately $1 \mathrm{MHz}$. This covers the lower range of harmonics of the switching frequency (in our case was $f_{\text {pwm }}=12.5 \mathrm{kHz}$ ), which produces most of the leakage current. This frequency limitation has been implemented to corroborate the validity of the model at low frequencies (The named band $\mathrm{A}, 9 \mathrm{kHz}$ to $150 \mathrm{kHz}$ ).

These sources have been transformed to frequency domain and they permit compare the model's response and carry out different conclusions: The validation of the model and divergences between simulation and experimental sources. The comparative of $I_{C M(j \omega)}=\left(I_{H G(j \omega)}+I_{C H G(j \omega)}\right)$ is shown in Fig.7. Where, it is formed by two figures: Fig.7 (a) shows the comparative between experimental results and simulation using simulated sources, in other hand Fig.7 (b) shows the same comparative but using real sources instead. Accuracy improves as expected when the experimental source is taken.

Fig. 7 shows the spectrum of $I_{C M}$ which its spectrum profile is formed by all odd and even coefficients. This is due because the duty cycle and amplitude of the perturbation source varies with time, therefore its shape varies and then its spectrum is spread along all the frequency range.

Notice, the spectrum coefficients amplitude of $I_{C M}$ is maintained throughout the frequency, namely due to two main reasons: firstly, the parasitic impedances are mainly formed by capacitive behavior which their values decrease
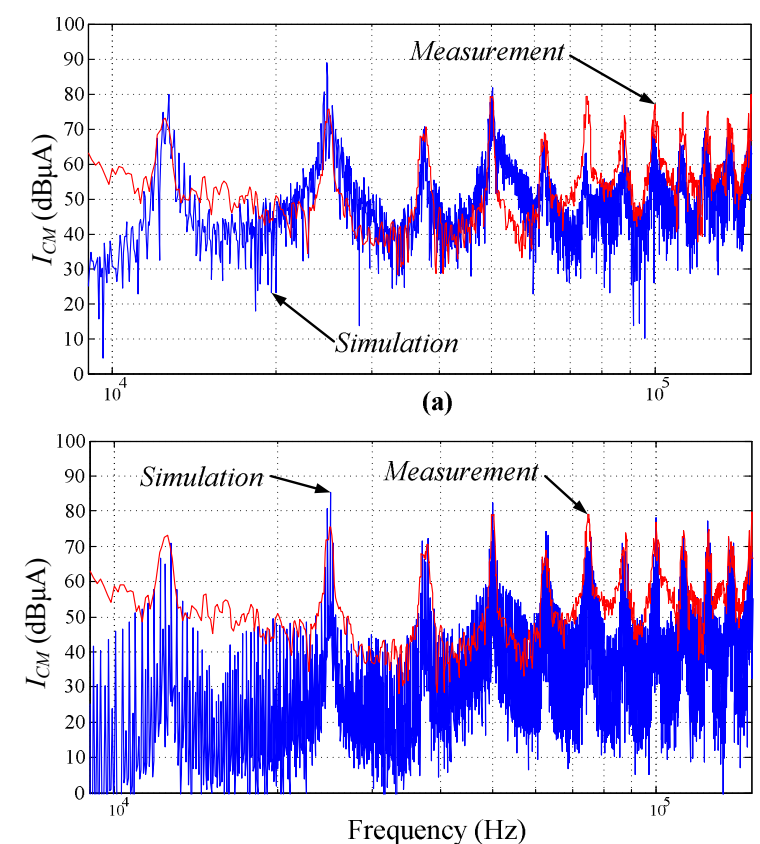

(b)

Fig. 7. Spectrum CM current ICM. (a) Voltage source disturbance from simulation. (b) Voltage disturbance source from experimental.

over the frequency and secondly the amplitude of the disturbance sources also decrease over the frequency. Therefore, the relation $I=\downarrow V \cdot j 2 \pi C \cdot \uparrow f$ maintains the amplitude of the current spectrum at the same level throughout the band A.

Finally, the simulation spectrum shows that the maximum amplitude which flows through the ground plane is not larger than $90 \mathrm{~dB} \mu \mathrm{A}(31.62 \mathrm{~mA})$ at $25 \mathrm{kHz}$. However, the maximum amplitude measured at $25 \mathrm{kHz}$ is $78 \mathrm{~dB} \mu \mathrm{A}(6.30 \mathrm{~mA})$. These divergences can be justified simulated sources do not take into account some MC characteristics which affects the MC behavior and its EMI source spectrum. In other hand when the measured sources are applied the current $I_{C M}$ is $84 \mathrm{~dB} \mu \mathrm{A}$ (15.8mA).

\section{CONCLUSIONS}

The combined time-frequency simulation method introduced in this paper allows a reasonable prediction of $\mathrm{CM}$ interferences produced by a MC with a low computational complexity.

Also, the comparative realized in this paper validate the good approximation of the EMI model, which improves when the experimental sources are used, reducing the existing differences from $12 \mathrm{~dB}$ down to $6 \mathrm{~dB}$, thus showing the need of certain refinements in the source model, in order to acquaint for the rise and fall times and for the commutation transient. It's important to notice that such transients might be obtained from a short time, low computational burden simulation, instead of measuring them from a prototype, thus allowing the prediction before the prototype construction. 
The extension of the method to higher frequencies is one of the objectives of future work. Also, the study of CM reduction applying different modulation strategies will be a research way to develop in future works.

\section{ACKNOWLEDGMENT}

The authors acknowledge the financial support received from "Ministerio de Ciencia e Innovación de España" for realizing this work under "TEC2007-61582" research project.

\section{REFERENCES}

[1] V. Jithesh and D. C. Pande, "A review on computational EMI modelling techniques," in Electromagnetic Interference and Compatibility, 2003. INCEMIC 2003. 8th International Conference on, 2003, pp. 159-166.

[2] R. Scheich and J. Roudet, "EMI conducted emission in the differential mode emanating from an SCR: phenomena and noise level prediction," Power Electronics, IEEE Transactions on, vol. 10, pp. 105-110, 1995.
[3] D. Gonzalez, et al., "New simplified method for the simulation of conducted EMI generated by switched power converters," Industrial Electronics, IEEE Transactions on, vol. 50, pp. 1078-1084, 2003.

[4] J. C. Crebier, et al., "A new method for EMI study in boost derived PFC rectifiers," in Power Electronics Specialists Conference, 1999. PESC 99. 30th Annual IEEE, 1999, pp. 855-860.

[5] F. Costa, et al., "Modeling of conducted common mode perturbations in variable-speed drive systems," Electromagnetic Compatibility, IEEE Transactions on, vol. 47, pp. 1012-1021, 2005.

[6] J. Balcells, et al., "EMI Generation Models for Switched Mode Power Supplies," The Smithsonian/NASA Astrophysics Data System ADS, vol. 416, pp. 421-426, 1998.

[7] L. Qian, et al., "Modular-Terminal-Behavioral (MTB) Model for Characterizing Switching Module Conducted EMI Generation in Converter Systems," Power Electronics, IEEE Transactions on, vol. 21, pp. 1804-1814, 2006

[8] S. Zhang and K. J. Tseng, "Modeling, simulation and analysis of conducted common-mode EMI in matrix converters for wind turbine generators," in Power Electronics and Motion Control Conference, 2008. EPE-PEMC 2008. 13th, 2008, pp. 2516-2523.

[9] J. F. Kolar, T. Krismer, F. Round, S, "The essence of three-phase AC/AC converter systems," Przeglad Elektrotechniczny, pp. 14-29, July 2008

[10] H. Akagi and T. Shimizu, "Attenuation of Conducted EMI Emissions From an Inverter-Driven Motor," Power Electronics, IEEE Transactions on, vol. 23, pp. 282-290, 2008.

[11] U. T. Shami and H. Akagi, "Experimental Discussions on a Shaft Endto-End Voltage Appearing in an Inverter-Driven Motor," Power Electronics, IEEE Transactions on, vol. 24, pp. 1532-1540, 2009. 